\title{
INFORMACIÓN Y TOMA DE DECISIONES: \\ OFICINAS DE ANÁLISIS INSTITUCIONAL EN UNIVERSIDADES CHILENAS
}

\section{Introducción ${ }^{2}$}

Este artículo analiza algunos aspectos centrales de la instalación y funcionamiento de unidades encargadas del procesamiento de información y apoyo a la toma de decisiones en las universidades chilenas. Genéricamente se les llama en esta investigación "oficinas de análisis institucional" (OAI).

Exigencias surgidas de manera espontánea, como consecuencia de la complejización del medio en que las instituciones deben desenvolverse y otras generadas por reparticiones estatales, han provocado que las unidades de análisis comenzaran a transformarse en instancias cada vez más relevantes en la gestión institucional.

Como es sabido, el desarrollo del sistema de educación superior chileno se ha caracterizado en los últimos años, entre otras cosas, por sus altos niveles de competitividad interinstitucional y bajos estándares de regulación. Esto lo ha llevado a aumentar significativamente sus grados de complejidad y, por lo mismo, a enfrentar fuertes desafíos de autorregulación.

En este escenario, el desarrollo de capacidades organizacionales para desatar autorreflexión y la generación de mecanismos de corrección de las desviaciones cobran especial importancia. Esto

2 Este artículo es el resultado del proyecto de investigación "Información y toma de decisiones en instituciones de educación superior. Estudio comparativo de unidades de análisis institucional en universidades chilenas", financiado por la Dirección de Investigación y Publicaciones de la Universidad Alberto Hurtado. En su primera etapa fue dirigido por Gastón Zamorano y contó con Guy Boisier como coinvestigador. La segunda etapa fue dirigida por Felipe Rivera y tuvo el apoyo del actual equipo de la Dirección de Aprendizaje Institucional. Su desarrollo final se hizo en colaboración con el Proyecto Anillos: "Programa de Investigación en Políticas de Educación Superior", financiado por CONICYT (Proyecto sOC01). 
implica formalizar y hacer fluida la conexión entre generación de información y toma de decisiones. La respuesta organizacional a estas demandas ha sido la creación de oficinas de análisis institucional.

Su instalación es una novedad en Chile y, por lo mismo, analizarlas en su constitución y funcionalidad resulta relevante para comprender los desarrollos organizacionales recientes y las potencialidades de autorregulación que están desarrollando las universidades chilenas.

\section{Las oficinas de análisis institucional}

\section{a) Consideraciones metodológicas}

La investigación que dio origen a este artículo fue realizada entre marzo y noviembre de 2007 y tuvo por objeto central analizar las experiencias de puesta en marcha y funcionamiento de las unidades de análisis institucional que recientemente han creado las universidades chilenas.

El estudio se realizó a partir de una selección de 13 universidades que cuentan con una OAI. La recolección de datos se hizo en dos niveles de profundidad. Por una parte, en una dimensión cualitativa, a través de entrevistas a seis directores de OAI. Por otra, se aplicó una encuesta a 34 directivos y profesionales de las 13 universidades. El trabajo de campo fue llevado a cabo entre julio y noviembre de 2007.

\section{b) Las OAI}

El primer paso de la investigación fue realizar un catastro de las OAI a través de la revisión de las páginas web de las instituciones. Al momento de la revisión, de las 61 universidades existentes en Chile, 48 tenían una repartición encargada del procesamiento de información y apoyo a la toma de decisiones. Se decidió llamarlas genéricamente "oficinas de análisis institucional", ya que ellas reciben diversos nombres dependiendo de la universidad. Los resultados del catastro se presentan en la tabla 1 . 
164 INFORMACIÓN Y TOMA DE DECISIONES: OFICINAS DE ANÁLISIS INSTITUCIONAL Felipe Rivera, Pablo Astudillo, Enrique Fernández

Tabla 1. Catastro de OAI en las universidades chilenas

\begin{tabular}{|l|l|c|c|}
\hline \multicolumn{2}{|l|}{ Universidad } & con OAI & sin OAI \\
\hline \multirow{2}{*}{ Consejo de Rectores } & Estatal & 16 & \\
\cline { 2 - 4 } & Privada & 9 & \\
\hline Privada & 23 & 13 \\
\hline Total & 48 & 13 \\
\hline
\end{tabular}

Fuente: elaboración propia

Junto con el procesamiento de información, las OAI tienen asociadas otras funciones. Éstas, sin embargo, varían significativamente entre las instituciones. En 32 casos estaban también a cargo del área de Planificación y Desarrollo, en 13 de la Autoevaluación y Aseguramiento de la Calidad y en 12 de la realización de Proyectos y Estudios.

Su dependencia jerárquica dentro de las universidades es diversa. De las 42 unidades para las que fue posible conseguir esta información en internet, la gran mayoría dependía directamente de la rectoría (25) y otro número importante de la vicerrectoría académica (8). En ocasiones obedecía a la prorrectoría (1), al consejo superior (1), a la vicerrectoría de Asuntos Económicos (1) o de Desarrollo (1). En un par de oportunidades la unidad constituía una vicerrectoría (2) o algún tipo de dirección autónoma (3).

\section{Surgimiento de las OAI}

El rápido crecimiento experimentado por el sistema universitario norteamericano en el periodo de posguerra obligó a los administradores de las universidades a dar cuenta pública del funcionamiento y desempeño de sus instituciones. Ello, a su vez, implicó invertir en sistemas de información que permitieran realizar una gran variedad de análisis y atender a diversos usuarios (Yorke, 2004). Este es el punto de partida de lo que actualmente se conoce como análisis institucional y que abarca una amplia gama de labores orientadas a informar la toma de decisiones y la planificación universitaria en áreas tales como admisión, planes de estudios, recursos humanos, asuntos estudiantiles, finanzas, instalaciones, entre otras. A partir de la década de 1990 esta tendencia comenzó a generalizarse, producto de la expansión e integración internacional de la educación superior. 
En el caso chileno, si bien una institución señaló que ya a fines de la década del 70 contaba con una unidad similar, dedicada a la planificación estratégica, las restantes OAI comenzaron a surgir recién en la segunda mitad de los años 90. Ellas constituyen una respuesta a la necesidad de contar con información para la toma de decisiones, en la medida en que el sistema de educación superior y la gestión institucional se fueron complejizando.

Este proceso de desarrollo de capacidades reflexivas y de autorregulación fue potenciado por exigencias externas, como las realizadas por el Programa MECESUP (1998), que pidió a las universidades contar con una planificación estratégica, tal como señalaron algunos de los entrevistados:

"Del 98 hasta el 2003, a partir de esa época todo el tema estratégico se comienza a colocar como tema fundamental. Aunque venía desde antes, porque el MECESUP te empieza a pedir planificación estratégica, nuestras primeras planificaciones estratégicas son del 2000. Se toma en consideración que no sólo es necesario tener esta Dirección de Estudios, porque es lo que se hacía, los estudios y los proyectos, pero ahora también había tomado la parte estratégica. Entonces se decide cambiarle el nombre en sentido de enfocarlo hacia un tema estratégico. En esa época había dos alternativas, colocarle análisis institucional, por institucional research, o colocarle un nombre que sería creado, entonces el rector dijo 'análisis institucional y desarrollo estratégico".

Un efecto similar tuvieron los procesos de acreditación liderados por la entonces Comisión Nacional de Acreditación de Pregrado, CNAP, que evidenciaron la necesidad de contar con unidades que centralizaran y procesaran información.

Como señaló un directivo:

"porque de todo lo que pedía la CNAP no teníamos nada de nada. Al principio nos demoramos ocho meses en juntar la información de una escuela”.

Uno de los objetivos explícitos de las OAI es hacer gestión de la información. Al respecto, se observa que muchas veces es el entorno 
de las instituciones el que las obliga a reorganizarlas en reiteradas ocasiones. Los actores son enfáticos en afirmar que ha sido en gran parte producto de la dinámica del sistema que las OAI se instalen como mecanismos de autorreflexión institucional.

Los directivos le asignan a las OAI un rol de tipo instrumental al interior de las universidades. Es decir, no se las concibe como unidades que lideren procesos, sino que las apoyan, ya sea a través de la información que proveen o de la coordinación de los procesos.

Como declarara uno de los directores entrevistados al preguntársele por la función de su unidad: hay que

"...tener muy claro que la información de la universidad debe estar dispuesta de manera tal que te pueda ser útil para estos procesos. Nosotros somos una unidad de servicios".

\section{Dificultades para la instalación de las OAI}

Dado que las OAI son unidades nuevas en las universidades, su principal problema es la legitimidad, es decir, alcanzar el reconocimiento necesario para realizar las funciones encomendadas e instalar determinadas operaciones en la organización.

Un ejemplo de estas dificultades lo constituye la centralización de la información para enviarla al entorno, tarea que habitualmente se le encarga a las OAI.

Como señaló una directora:

"...esa fue una lucha que nosotros dimos, no sólo yo como directora, sino que todos los directores. De validarnos como informadores oficiales, porque antes todos querían dar sus datos de matrícula. Nos validamos no a partir de un proceso impositivo, sino que a través de una suerte de seducción: 'tú tienes que entregar los datos de matrícula, para qué te vas a hacer problemas contando chiquillos, no es tu pega, yo te lo hago"'.

El problema de la legitimidad de las unidades tenía relación no sólo con esta suerte de monopolio informativo, sino también con 
el propio carácter de los procesos que las OAI intentan instalar en la organización:

"cuando partimos, hablar de autoevaluación, hablar de acreditación, hablar de planificación estratégica, había gente que se le paraban los pelos, había algunos que se negaban a hablar de la planificación estratégica. 'Qué tengo que estar implementando procesos de la empresa privada acá, si esta no es una fábrica de parkas'. O los académicos formulando proyectos para traer recursos a la universidad, "pero si yo soy académico, por qué me meten en esto"'

En ese mismo sentido, un desafío para las OAI ha sido lograr la definición de un perfil organizacional, que tenga aceptación en el entorno en que les toca desenvolverse:

"algo que queremos romper es esa visión de los departamentos o direcciones de evaluación y estudio que son unas entidades burocráticas, llenas de números que no sirven para nada, con 'turros' de información que no sabes por dónde partir, eso es lo que no quisiéramos".

Las formas en que se ha enfrentado el problema de la legitimidad al interior de la organización han sido variadas. Algunas unidades, como se afirma en la cita inicial de este apartado, lo han hecho por la vía de la "seducción", al aparecer liberando de trabajo a los académicos y directivos.

En otras ocasiones, el apoyo de las autoridades superiores ha sido clave para dotar de peso político a las OAI. Finalmente, algunos han usado estrategias de cooptación de ciertos líderes de la institución:

"yo creo que eso es gradual y va dependiendo de cómo tú vas identificando ciertos actores, ciertos líderes naturales, que son los que tienen que ir sumando, los vas sumando y vas haciendo causa común. Y se van incorporando de a poco y al final se permea toda la unidad, pero es un proceso. Ahora el tema es cómo tú (...) vas ganando adeptos a tu gestión y vas trabajando con los líderes naturales". 


\section{El entorno: complejización y competitividad}

Las OAI son un importante punto de contacto entre las universidades y su entorno. Esta relación se da en dos sentidos. Primero, en el ya mencionado: la OAI provee de información oficial a las instancias externas que lo requieran; segundo, provee a la organización de información respecto del entorno en que ésta se desenvuelve.

Los actores coinciden en que la expansión y complejización del sistema de educación superior chileno les impone una permanente observación de éste, con el fin de generar la información necesaria para la toma de decisiones. Por lo mismo, una tarea fundamental de las OAI -declaran- es desarrollar la capacidad de observar a la competencia, tanto para realizar comparaciones de indicadores de gestión como para determinar sus desarrollos efectivos y potenciales.

Como señalara uno de los entrevistados: "uno de los temas nuestros es el tema estratégico, siempre estamos pendientes de lo que está pasando. Hoy en día, todas las empresas están en eso, es la única manera en que tú puedas ser exitoso, debes estar pendiente de lo que pasa afuera".

En la investigación se pudo constatar que uno de los focos principales de observación que hacen las OAI está puesto en la oferta y demanda de carreras. Esto, sin duda, obedece a la intensa competencia en el sistema de educación superior por captar alumnos nuevos y aumentar la selectividad.

Pero la observación de la competencia no sólo se restringe al análisis de datos generales, sino que abarca también al contenido. En ocasiones, como lo muestra la cita consignada a continuación, para poner en sintonía los propios desarrollos con otros considerados más exitosos:

"a veces hay temas que nosotros observamos, como por ejemplo, te invento, arqueología. Nos damos cuenta de que nuestra carrera estaba demasiado orientada no sé a qué, mientras que nuestra competencia estaba orientada a tal lado, entonces se dice ¿por qué no revisan este tema? 
¿Por qué no hacen un giro de cómo este tema calzaría mejor con los alumnos? Lo estudian, lo desarrollan, lo arman y lo traen".

\section{Aprendizaje y desarrollo estratégico}

Otros dos elementos, interrelacionados entre sí, se reiteran frecuentemente en las OAI. Se trata de los procesos de aprendizaje organizacional y de desarrollo estratégico.

Los aprendizajes organizacionales son de distinto orden. Algunos tienen por objetivo simplemente conocer aspectos del funcionamiento interno de la universidad, otros optimizar determinadas operaciones, avanzar en la "excelencia" o desarrollar capacidades para realizar algunas tareas.

Como señaló uno de los entrevistados al preguntársele por las áreas en que esto se puede apreciar:

"entonces, tú vez que hay unos temas que se repiten en todas las carreras, no hay seguimiento de egresados. Entonces, nos ganamos un FDI y ahora estamos levantando para toda la universidad un sistema de seguimiento de egresados. Hay temas que son comunes y temas que son particulares, y tú vas nivelando y aquí viene el tema de aprendizaje organizacional".

Los entrevistados señalan que la instalación no ha sucedido de manera automática en la organización, sino que ha sido un proceso que ha demandado bastante tiempo y trabajo. Sin embargo, observan que el aprendizaje organizacional se ha ido consolidando:

"yo creo que hay aprendizaje, hay tareas aprendidas, por lo menos uno se percata que cuando se mira dos veces una cosa, ya es un logro importante. De hecho, se instaló una especie de reflexión de lo que se hace".

Al analizar los resultados de la encuesta en el ítem correspondiente a las condiciones que se dan para el desarrollo de procesos de aprendizaje institucional en las universidades, las propias OAI hacen un diagnóstico bastante positivo, como se aprecia en la tabla 2: 
170 INFORMACIÓN Y TOMA DE DECISIONES: OFICINAS DE ANÁLISIS INSTITUCIONAL Felipe Rivera, Pablo Astudillo, Enrique Fernández

Tabla 2. ¿Cuán de acuerdo está usted con las siguientes afirmaciones?

\begin{tabular}{|l|c|c|c|c|c|c|c|}
\hline \multirow{2}{*}{ Afirmación } & \multicolumn{3}{|c|}{ de acuerdo } & \multicolumn{3}{c|}{ en desacuerdo } & $\mathrm{n} / \mathrm{s}$ \\
\cline { 2 - 9 } & Total & m.d.a. & d.a. & e.d. & m.e.d. & Total & $\mathrm{n} / \mathrm{r}$ \\
\hline & 25,8 & 3,2 & 22,6 & 61,3 & 6,5 & 67,8 & 6,5 \\
\hline $\begin{array}{l}\text { En esta institución los errores son } \\
\text { considerados parte del proceso }\end{array}$ & 74,2 & 6,5 & 67,7 & 22,6 & 0 & 22,6 & 3,2 \\
\hline $\begin{array}{l}\text { En esta institución se procura } \\
\text { aprender de los errores, sin necesidad } \\
\text { de buscar responsables directos }\end{array}$ & 80,7 & 6,5 & 74,2 & 16,1 & 0 & 16,1 & 3,2 \\
\hline $\begin{array}{l}\text { El trabajo que se realiza desde mi } \\
\text { unidad está enfocado en generar una } \\
\text { cultura de "prueba - aprendizaje", } \\
\text { que permite efectuar exploraciones } \\
\text { estratégicas }\end{array}$ & 67,8 & 32,3 & 35,5 & 29,0 & 0 & 29,0 & 3,2 \\
\hline
\end{tabular}

$\mathrm{n}=34$

Los procesos de aprendizaje organizacional están estrechamente ligados a la planificación estratégica: otra de las funciones que habitualmente asumen las OAI. Los entrevistados coinciden en que ambos constituyen un círculo: "entonces tenemos los diagnósticos que dan origen a los planes, y los planes más el diagnóstico, al aprendizaje organizacional".

Para una adecuada planificación y desarrollo estratégico de la universidad se considera fundamental el monitoreo constante del quehacer institucional, pero también del entorno.

Ante la pregunta acerca de cuáles son las funciones principales que la OAI desempeñan en este sentido, los encuestados respondieron lo siguiente:

a) comprender estadísticamente la institución (87\%);

b) recolectar, analizar y compartir información con las demás unidades de la organización (87\%);

c) analizar el entorno, tanto en lo referido a las demás instituciones como al desarrollo general del sistema (77\%);

d) resguardar el cumplimiento de la misión institucional (67\%);

e) gestionar ideas, propuestas y resolución de problemas (64\%), y

f) poner a su institución en escenarios posibles, permitiendo a las autoridades anticiparse a lo que sucederá (61\%). 
La planificación estratégica es considerada por los encuestados como un instrumento fundamental al momento de tomar decisiones relacionadas con el desarrollo futuro de la universidad. De igual modo, se considera que ella debe contener y representar adecuadamente los intereses y características de la propia institución, lo que a su vez es señal de la importancia asignada al conocimiento y aprendizaje institucional (tabla 3).

Tabla 3. ¿Cuán de acuerdo está con las siguientes afirmaciones? (en porcentajes)

\begin{tabular}{|l|c|c|c|c|c|c|c|}
\hline \multirow{2}{*}{ Afirmación } & \multicolumn{3}{|c|}{ de acuerdo } & \multicolumn{3}{c|}{ en desacuerdo } & $\mathrm{n} / \mathrm{s}$ \\
\cline { 2 - 9 } & Total & m.d.a. & d.a. & e.d & m.e.d. & Total & $\mathrm{n} / \mathrm{r}$ \\
\hline $\begin{array}{l}\text { La planificación estratégica es una } \\
\text { herramienta útil, que considera la forma en } \\
\text { que se hacen las cosas en mi institución }\end{array}$ & 90,3 & 54,8 & 35,5 & 6,5 & 0 & 6,5 & 3,2 \\
\hline $\begin{array}{l}\text { La planificación estratégica es considerada } \\
\text { cada vez que se debe decidir algo }\end{array}$ & 77,5 & 45,2 & 32,3 & 12,9 & 6,5 & 19,4 & 3,2 \\
\hline
\end{tabular}

$\mathrm{n}=34$

\section{Información: valor público y toma de decisiones}

Tanto los directivos entrevistados como quienes participaron en las encuestas destacan el valor de la información y, por lo mismo, del papel de sus propias unidades. La importancia que le asignan trasciende a los requerimientos de gestión, llegando hasta una cuestión de orden político: la información, en el sistema de educación, tiene valor público. Los actores distinguen esta característica como fundamental en dos sentidos: en lo relativo a la necesidad de proveer a los usuarios de información consistente y oportuna, y en disponer institucionalmente de datos del sistema de educación superior.

En efecto, los actores identifican como un déficit la falta de sistemas de información nacional que alimenten la toma de decisiones institucionales.

Los directivos resaltan que, respecto de la toma de decisiones institucionales relevantes, la información juega un papel cada vez mayor en las instancias de gobierno y gestión. 
Como señalara un entrevistado:

"por ejemplo, en el Consejo Académico, yo creo que algo bien importante y bien destacable de nuestra universidad, es que en un tiempo no menor la universidad está tomando decisiones bastante informadas. Es una diferencia con lo que ocurría hace varios años, en los que las decisiones eran muy por el 'feeling' que tenían las personas, en el proceso de la toma de decisiones. Por ejemplo, en los últimos días una facultad presentó un proyecto para venir con una carrera acá a Santiago. Y el mismo Consejo tomó la decisión de que la unidad de planificación emitiera una opinión, en términos de validar el informe, sobre todo las proyecciones económicas que habían hecho los colegas, como también ver cómo eso se marcaba con el plan estratégico. Nuestro estudio reflejaba que no era una buena alternativa, que además no estaba asociado con la matriz de crecimiento de la universidad (...) Entonces el Consejo Académico, que perfectamente podría haber dicho: 'esto es importante, no nos enojemos con ellos, dejémoslos que prueben', profesionalizó el tema de la toma de decisiones, acompañándose por la información. Finalmente se planteó lo que nosotros dijimos".

Las OAI están comenzando a transformarse en un apoyo fundamental para la toma de decisiones institucionales:

"el objetivo clave es poder alimentar las decisiones que toma rectoría (...) O sea yo no me pongo a redactar un artículo, básicamente es una tabla con la información clave que se necesita para la toma de decisiones".

En esa tarea las OAI concentran sus esfuerzos:

"nosotros nunca hemos sido una institución, con grandes planes de desarrollo, o un master plan que lo sigamos año a año o mes a mes, en el cual tengamos 70 indicadores. Estamos trabajando en esa línea también, pero la gran fuerza está en disponer de buena información para que los buenos liderazgos de las distintas áreas puedan tomar decisiones".

Producto de la importancia que asignan a esta tarea, quienes integran las OAI comienzan a enjuiciar la eficiencia y velocidad 
con que son tomadas las decisiones para las que ellos proveen la información. Al preguntársele respecto a esto, las respuestas fueron las siguientes:

Tabla 4. Teniendo en cuenta las 3 últimas decisiones importantes tomadas en su institución, usted diría que... (en porcentajes)

\begin{tabular}{|l|c|c|c|c|c|c|c|c|}
\hline \multirow{2}{*}{ Afirmación } & \multicolumn{3}{|c|}{ de acuerdo } & \multicolumn{3}{c|}{ en desacuerdo } & n/s \\
\cline { 2 - 8 } & Total & m.d.a. & d.a. & e.d. & m.e.d. & Total & n/r \\
\hline Fueron acertadas, ya que fueron discutidas & 93,7 & 48,5 & 45,2 & 3,2 & 0 & 3,2 & 3,2 \\
\hline Fueron tomadas a la velocidad adecuada & 67,7 & 12,9 & 54,8 & 29,0 & 0 & 29,0 & 3,2 \\
\hline Fueron bien ejecutadas & 80,7 & 22,6 & 58,1 & 16,1 & 0 & 16,1 & 3,2 \\
\hline $\begin{array}{l}\text { Estuvieron involucradas las personas } \\
\text { correctas y de la manera correcta }\end{array}$ & 83,9 & 22,6 & 61,3 & 12,9 & 0 & 12,9 & 3,2 \\
\hline $\begin{array}{l}\text { Se basaron las decisiones en la información } \\
\text { adecuada }\end{array}$ & 87,1 & 25,8 & 61,3 & 9,7 & 0 & 9,7 & 3,2 \\
\hline $\begin{array}{l}\text { Los que tomaron decisiones provenían de } \\
\text { los niveles adecuados }\end{array}$ & 87,1 & 51,6 & 35,5 & 9,7 & 0 & 9,7 & 3,2 \\
\hline $\begin{array}{l}\text { Los incentivos de la organización fueron un un } \\
\text { estímulo para que las personas tomaran la } \\
\text { decisión correcta }\end{array}$ & 74,2 & 16,1 & 58,1 & 16,1 & 3,2 & 19,3 & 6,5 \\
\hline
\end{tabular}

$n=34$

Un porcentaje de respuesta llama particularmente la atención. Se refiere al juicio de los actores sobre la velocidad con que son tomadas las decisiones. Un 29\% estima que éstas no son tomadas a la velocidad adecuada.

Algunos entrevistados identificaron, sin embargo, una dificultad importante para proveer a las autoridades de información de calidad para la toma de decisiones: los sistemas institucionales de información.

Como señalaran:

"de pronto demasiadas veces tienes que recurrir a unidades, porque no está la información de llegar y sacar. Sí, recurrimos más de lo que deberíamos, ya que deberíamos tener mejores sistemas de información".

Incluso consideran que:

"el desafío es mejorar los sistemas de información, optimizar mucho más". 


\section{Las OAI como mecanismos de autorreflexión organizacional, acoplamiento al entorno y corrección de desviaciones}

Una investigación desarrollada por F. J. Volkwein en 1999 (citado en Yorke, 2004) clasifica las funciones y competencias de las OAI de acuerdo con las audiencias a que están dirigidas (interna vs. externa) y al papel principal o perfil que cumplen dentro de la organización (administrativo vs. académico). Esto permite identificar las funciones de las OAI con relación a audiencias externas, cuando se trata de mecanismos de acoplamiento al entorno, e internas, cuando se trata de mecanismos de corrección de desviaciones (tabla 5).

Tabla 5. Funciones de OAI de acuerdo con audiencias y roles

\begin{tabular}{|c|c|c|}
\hline \multicolumn{3}{|c|}{ Institutional Research Typology(Volkwein) } \\
\hline & \multicolumn{2}{|c|}{ AUDIENCIA } \\
\hline & Interna & Externa \\
\hline \multirow{2}{*}{ 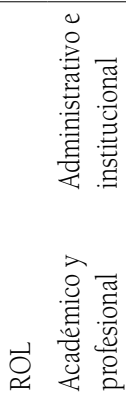 } & $\begin{array}{l}\text { Provisión de información para propósitos } \\
\text { administrativos y de gestión. Foco en la } \\
\text { búsqueda y análisis de información. } \\
\text { Generación de reportes apropiados para } \\
\text { los que toman decisiones }\end{array}$ & $\begin{array}{l}\text { Presentación de la institución a agentes } \\
\text { externos. Foco en el ajuste con los } \\
\text { requerimientos para la acreditación y/ } \\
\text { para obtener financiamiento externo. } \\
\text { Supone capacidad de análisis político. }\end{array}$ \\
\hline & $\begin{array}{l}\text { Generación de nueva información y } \\
\text { evaluar y contrastar los datos internos. } \\
\text { Formulación de recomendaciones para la } \\
\text { toma de decisiones. Implica capacidades } \\
\text { técnicas y estratégicas }\end{array}$ & \begin{tabular}{|l|} 
Foco en la generación de información \\
para fines académicos, especialmente \\
calcular indicadores o compararse con \\
otras instituciones. No entregar feedback \\
para la gestión interna.
\end{tabular} \\
\hline
\end{tabular}

Fuente: Yorke 2004, p. 147.

Las OAI estudiadas están presentes en cada uno de los cuadrantes de la tipología propuesta por Volkwein, ya que cumplen funciones como procesamiento de información, planificación y desarrollo, proyectos y estudios y autoevaluación y aseguramiento de la calidad.

Estas oficinas se instalan como mecanismos que cumplen, entonces, varias funciones. Una de las más relevantes es la reducción de complejidad, ya que trabajan recopilando, analizando y sistematizando información que las instituciones y el propio sistema requieren para operar. 
Es posible afirmar que las OAI se transforman en espacios donde tiene lugar una autorreflexión organizacional que opera, al menos, en dos sentidos: a) como mecanismos de acoplamiento al entorno, y b) como mecanismo de corrección de desviaciones.

El primer caso se refiere al hecho de que las OAI proveen los insumos para que la organización no sólo logre una comprensión del entorno, sino para que se acople a él a través de procedimientos rutinarios que éste posee (como entrega de información), se ajuste a normas (como los procesos de acreditación) y se adecue a los cambios contingentes generados por la propia dinámica del sistema (como, por ejemplo, aumento de la oferta de programas, nuevos fondos concursables, entre otros).

En el segundo caso, la corrección de desviaciones se desarrolla internamente. Al realizar una observación de la propia organización, están evaluando en forma permanente el grado en que están cumpliendo sus propios propósitos organizacionales. Es probable que, por lo mismo, en varias ocasiones hayan asumido además las funciones de planificación. De esa manera, se constituyen en un mecanismo de corrección de desajustes y desviaciones respecto de los propósitos institucionales.

Evidentemente, esto sólo es posible en la medida que la universidad cuenta con una planificación o lineamientos que fijen propósitos contra los que las OAI puedan contrastar las operaciones y desarrollos.

Es posible afirmar que las OAI son mecanismos característicos de sistemas autónomos, capaces de autorregularse. Operan generando reflexión e impulsando desarrollos, con el objeto de responder a la complejización creciente del sistema.

En síntesis, las OAI funcionan reduciendo complejidad, permitiendo a las universidades proyectar su futuro, manteniendo el equilibrio entre las exigencias del entorno y los intereses planificados de la organización. En otras palabras, dotan a las organizaciones de las capacidades necesarias para funcionar en un entorno de complejidad creciente. 


\section{Conclusiones}

Hemos presentado los principales resultados del estudio sobre la instalación y funcionalidad de las OAI en las universidades chilenas. Si bien éstas son de creación reciente y tienen algunos problemas de legitimidad al interior de las universidades, del estudio se desprende su progresiva importancia para estas instituciones.

Las OAI no sólo facilitan el acoplamiento de las universidades a un entorno cada vez más complejo, sino que proveen los insumos para desatar procesos de autorreflexión y corrección de desviaciones con relación a los objetivos planificados por éstas. La reducción de complejidad se hace posible por la vía de la recolección, análisis y sistematización de información de la propia universidad y del sistema en general.

En este punto, sin embargo, las OAI se encuentran en ocasiones no poco frecuentes con la dificultad generada por las carencias de sistemas de información que la provean en línea y actualizada. Algo similar ocurre con la precariedad de los sistemas de información sobre educación superior en el país.

La proliferación y consolidación de las OAI en las universidades chilenas es una señal inequívoca de un aumento de la profesionalización de la gestión institucional, que busca tomar decisiones cada vez mejor informadas y no sólo desde las apreciaciones personales de los involucrados.

No obstante, a pesar de que su funcionalidad organizacional aparece como obvia, su instalación no ha estado exenta de problemas. Ello se deriva de que es un tipo de unidad nueva que se apropia de operaciones antes radicadas en unidades académicas o administrativas, o de que intenta instalar procesos que los actores no vinculan fácilmente con las funciones tradicionales de la universidad. Esto les genera un problema de legitimidad que han intentado solucionar por distintas vías.

Resulta probable, además, que algunas de las OAI no sean mucho más que una respuesta formal a requerimientos internos y 
externos, o una emulación de estrategias de desarrollo observadas en otras universidades consideradas exitosas, y que, por consiguiente, no hayan sido provistas ni de los recursos ni de la autoridad necesaria para asumir en forma efectiva las funciones que se le asignan.

Como se planteó al comienzo de este artículo, el sistema de educación superior chileno está sometido hoy a fuertes presiones por aumentar sus niveles de autorregulación. Las OAI son una respuesta organizacional a este requerimiento. Si bien ellas constituyen respuestas particulares y parciales, a las cuales subyace además el principio de hacer a sus propias universidades más competitivas, desatan procesos de autorreflexión y correcciones de desviaciones. En ese sentido, es esperable que, en su operatoria de conjunto, contribuyan de manera significativa a la autorregulación de la totalidad del sistema de educación superior.

Agradecemos la participación en el estudio de las oficinas de análisis institucional de las siguientes universidades: Austral de Chile, Católica de la Santísima Concepción, Católica de Temuco, Pontificia Católica de Valparaíso, Católica del Maule, de la Frontera, de Playa Ancha, de Talca, de Valparaíso, del Desarrollo, Diego Portales, Iberoamericana de Ciencia y Tecnología y Técnica Federico Santa María.

\section{Referencias bibliográficas}

Beer, Stafford (1981) Brain of the Firm. 2a edición. Londres: John Wiley.

Beer, Stafford (1985) Diagnosin the System for Organizations. Chichester: John Wiley.

Brunner, José Joaquín y Elaqua, Gregory (2003) Informe Capital Humano en Chile. Santiago de Chile: Universidad Adolfo Ibáñez.

Cáceres, Carlos (2007) Planeación estratégica en universidades del Consejo de Rectores: evidencias del periodo 2000-2005. Calidad en la Educación, 27, pp. 108-150.

Consejo Nacional de Innovación para la Competitividad (2006) Informe Final. Santiago de Chile: Consejo Nacional de Innovación para la Competitividad.

Luhmann, Niklas (2005) Organización y decisión. Autopoiesis, acción y entendimiento comunicativo. Barcelona: Anthropos. 
Maturana, Humberto y Porksen, Bernhard (2004) Del ser al hacer. Los Orígenes de la Biología del Conocer. Santiago de Chile: LOM Ediciones.

Nonaka, Ikujiro y Takeuchi, Hirotaka (1999) La organización creadora de conocimiento. Cómo las compañias japonesas crean la dinámica de la innovación. México: Oxford University Press.

Rodríguez, Darío (1992) Gestión Organizacional. Santiago de Chile: Editorial Pontificia Universidad Católica de Chile.

Rojas, Gustavo (2005) Modelos Universitarios. Los rumbos alternativos de la universidad y la innovación. México: Fondo de Cultura Económica.

Villa Sánchez, Aurelio; Escotet, Miguel y Goñi, Juan José (2007) Modelo de Innovación de la Educación Superior. Bilbao: Ediciones Mensajero, Universidad de Deusto.

Von Bertalanffy, Ludwig (2002) Teoría General de los Sistemas. México: Fondo de Cultura Económica.

Yorke, Mantz (2004) Institutional research and its relevance to the performance of higher education institutions. Journal of Higher Education Policy $\&$ Management, pp. 141-152.

Recibido: 17 de septiembre de 2008 Aceptado: 24 de marzo de 2009 